\title{
Early Breast Cancer Tumor Detection on Mammogram Images
}

\author{
Naishil N. Shah \\ Computer Engineering, \\ Dharmsinh Desai University, \\ Nadiad-387001
}

\author{
Tushar V. Ratanpara \\ Asst. Professor, \\ Department of Computer Engi- \\ neering, \\ Dharmsinh Desai University, \\ Nadiad-387001
}

\author{
C.K.Bhensdadia \\ Professor, \\ Department of Computer Engi- \\ neering, \\ Dharmsinh Desai University, \\ Nadiad-387001
}

\begin{abstract}
This paper describes a system that enhances the mammogram to detect the possible lesion or tumor. First the pre-processing task is performed on the mammogram. The pre-processing phase has been sub divided into 4 parts to get the Region of interest and remove the external factors that affect the system in obtaining the possible lesion. Then the segmentation process is performed on the mammogram to get only those portions of the image which are greater than a particular predefined threshold value. After segmentation the edge detection process is performed on this segmented mammogram to display edges over masses. Morphological filtering is done to get masses highlighted with a border. Out of these masses the maximum probable mass that could be a lesion/tumor is highlighted. Finally this mass detected clear border mammogram image is superimposed over the original mammogram to enhance the mass from the mammogram.
\end{abstract}

\section{General Terms}

Breast cancer tumor detection, Mammogram image processing.

\section{Keywords}

Breast cancer tumor, Feature extraction, Gray-scale, Image processing, Image enhancement, Image segmentation, Image edge detection, Image processing, Mammogram, Morphological operations, Thresholding

\section{INTRODUCTION}

Breast cancer is the most common cancer among women in the United States, other than skin cancer. It is the second leading cause of cancer death in women, after lung cancer. Detection and diagnosis of breast cancer in its early stage increases the chances for successful treatment and complete recovery of the patient .The three major ways to screen breast cancer are self-examination, clinical examination, and mammography. Screening mammography is currently the best available radiological technique for early detection. A mammogram is an $\mathrm{x}-$ ray of the breast. The goal of screening exams for early breast cancer detection is to find cancers before they start to cause symptoms. There are so many techniques that have been developed for the detection of masses and bilateral asymmetry. For enhancement the techniques are Gaussian filter, Adaptive histogram equalization and Wavelet fusion [1]. Masses are basically high frequency components in the mammogram. Masses are detected using Multi-scale blob detection or by Segmentation operations and also by using bilateral asymmetry technique [2].Bilateral asymmetry was detected using Single image comparison (SIC) and Multiple Image Comparison [3]. Some segmentation are classified into one of two groups, supervised or unsupervised [4].Object detection has been done using Generalized Hough Transform (GHT) [5]. In particular, wavelet orthogonal and biorthogonal bases have been successfully used in many applications. It has already been stressed that the lack of translation invariance of these representations can be a severe drawback because this leads to the introduction of a large number of artifacts in the reconstruction after the processing of the wavelet coefficients [6].However the detection of small malignancies is especially difficult in younger women who tend to present denser breast tissues. Since the calcification have high attenuation properties and small dense tissues similar to bones, they tend to present low contrast. Thus their visual screening is difficult for physicians [7]. Figure 1 contains proposed approach for early best cancer tumor detection.

\section{PROPOSED APPROACH OF EARLY BREAST CANCER TUMOR DETEC- TION}

In this paper tasks are divided into two parts. First task is Pre-processing and the second is the image segmentation. In the pre-processing task first the mammogram is enhanced and then noise reduction is performed. After that the label is removed and lastly the pectoral muscle is removed. First the pre-processing tasks are performed on the mammogram in order to remove the external noise, label and pectoral muscle from the mammogram. For image segmentation, contrast improvement and grayscale thresholding techniques are applied for enhancing the whole image. Then, the segmentation process is performed on the mammogram to get only those portions of the image which are greater than a particular predefined threshold value. Then, Edge detection is performed on this segmented mammogram to display edges over masses. On this edge detected mammogram morphological filtering method is applied to obtain clear and distinct masses. Out of these masses the maximum probable mass that could be a lesion/tumor is detected. Finally this mass detected clear border mammogram image is superimposed over the original mammogram to enhance the mass from the mammogram. 


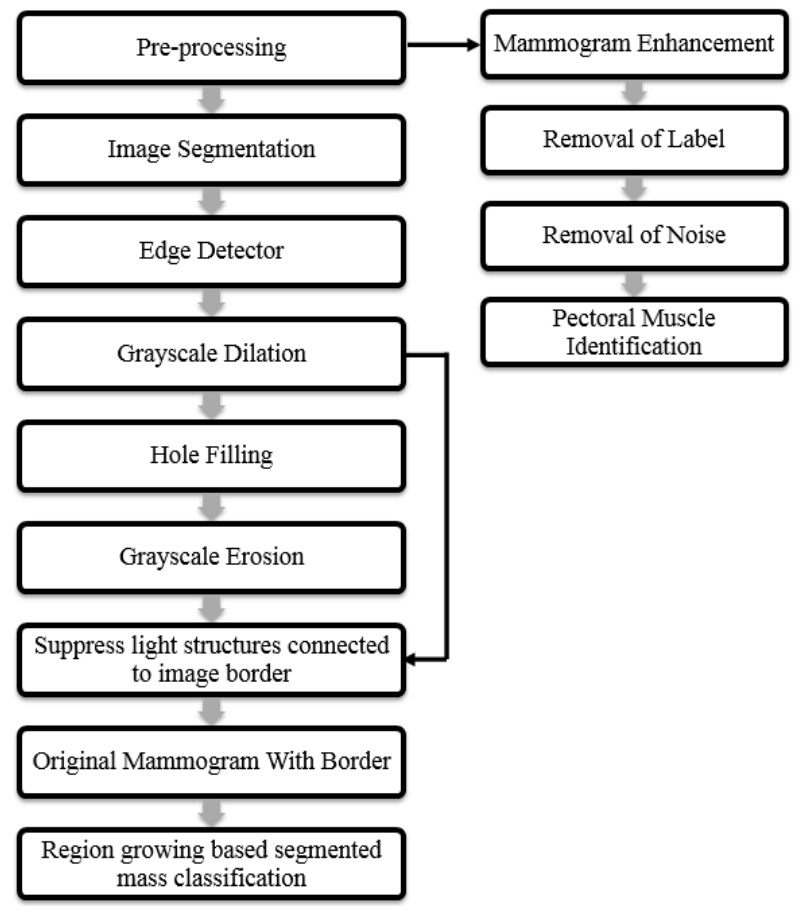

Fig. 1. Proposed Appraoch

\subsection{Pre-Processing}

In the pre-processing stage, first the input image is enhanced using histogram equalization and normalization (mammogram Image) and then it is segmented to limit the search for unwanted area from the background of the mammograms in order to improve the quality of the image and reduce noise. The unwanted areas are labels on the mammogram and pectoral muscles on the mammogram. The pre-processing phase has been sub divided into 4 different parts as follows:

\subsubsection{Mammogram Enhancement}

For enhancing the mammogram histogram equalization and normalization technique is used. Histogram equalization is a method that adjusts the contrast using the image's histogram. This method adjusts, the intensities distributed on the histogram perfectly. This method helps to convert the areas of lower local contrast to gain a higher contrast. Histogram equalization accomplishes this by effectively spreading out the most frequent and the most common intensity values. This method is useful in images with backgrounds and foregrounds that are both bright or both dark. Using this it gives the best enhancement in Mammogram and X-rays images.

\subsubsection{Removal of Label}

Four different type of noise can be found that appears in these mammogram images as shown in figure 2 :

a) High intensity label.

b) Low intensity label.

c) Scanning artifact.

d) Tape artifact. The following figure shows an example of each one:

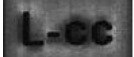

(a)

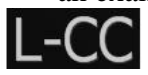

(b)

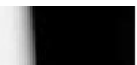

(c)

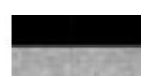

(d)

So the objective in this step is to remove any of these from the mammogram images if they exist. But here in this paper the focus is only on Low intensity label. The label present in the mammogram image is removed by using the blob / bounding box analysis technique. Breast Orientation: Depending upon the density value, it is determined whether the mammogram is of L-MLO (Left Mediolateral Oblique) view or the R-MLO (Right Mediolateral Oblique) view. If the mammogram is of the R-MLO view then the label is removed using Blob / Bounding Box analysis technique and if the mammogram is of the L-MLO view then first it is converted into its mirror image and then the label is removed using Blob / Bounding Box analysis technique as shown in figure 2 .

\subsubsection{Removal of Noise}

The noise in the mammogram is reduced using median filtering. Median filtering is as same as an averaging filter, in that each output pixel is set to an average of the pixel values in the neighborhood of the corresponding input pixel. Here, the value of an output pixel is determined by the median of the neighborhood pixels, rather than the mean. The median filter is better than the mean filtering. Median filtering is less sensitive therefore it is used to remove these outliers without reducing the 'sharpness of the image.

\subsubsection{Pectoral Muscle Identification}

The pectoral muscle has to be removed from the breast region so that only the specific region of interest (ROI) is considered. The pectoral muscle contains high intensity pixels which might affect the mass detection process so this portion has to be removed from the mammogram so that the concentration is mainly on the region where the possibility of lesion/tumor is maximum. The pectoral muscle is removed by using Histogram based region isolation shifting technique. Here pectoral muscle is detected using thresholding technique. In this case, the straight line separates the muscle from the breast and a mask is applied to separate the image from it. Table 1 shows preprocessing tasks of five patients.

Table 1. Pre-Processing tasks on five patients on mammogram images

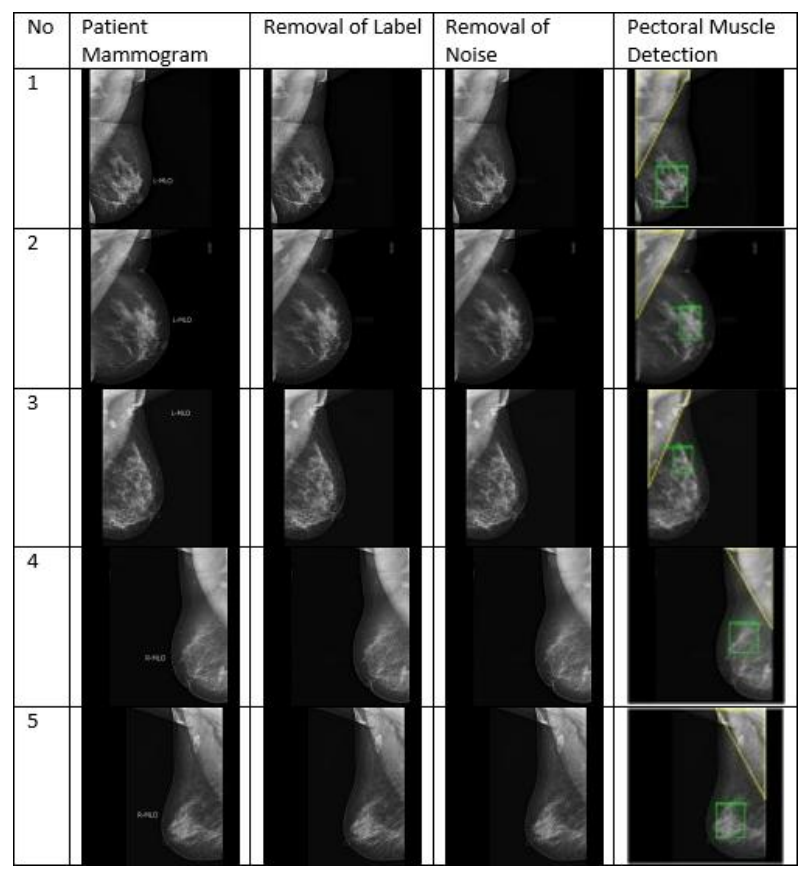




\subsection{Segmentation}

\subsubsection{Image Segmentation}

Segmentation can be used many ways but here segmentation has been performed based on segmentation of masses by using thresholding technique as shown in figure 3 . In thresholding technique it converts a gray-scale image into a binary image. Image segmentation is typically used to find objects, lines, curves and boundaries in images. More precisely, image segmentation is the process in which the objects can be easily identified. In segmentation it is partitioning a digital image into multiple segments. Seed point $\mathrm{S}$ is chosen as highest intensity value in the mass and threshold value $\mathrm{T}$ is chosen by looking at its histogram.
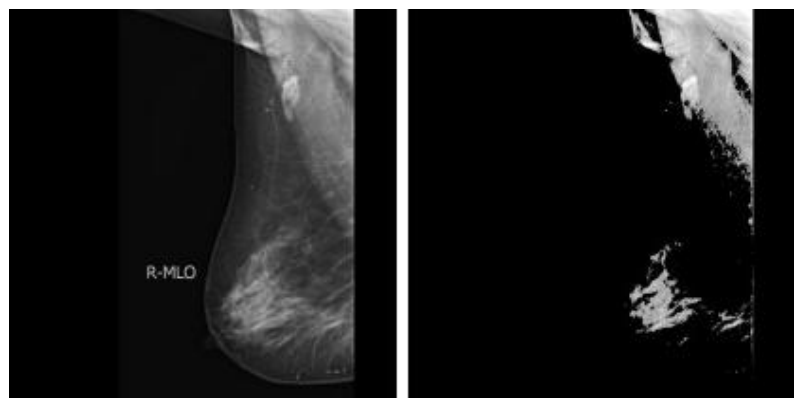

Fig. 3.(a) Normal mammogram image(b) Segmented mammogram image

\subsection{Edge Detector}

There are many edge detection techniques available like Gradient based Edge Detection, Laplacian based Edge Detection, Sobel Operator, and Robert's cross operator, Prewitt's operator, Laplacian of Gaussian etc. canny edge detection technique is used in this approach.

\subsubsection{Canny Edge}

Canny edge detection is used to extract the border. This optimal edge detector can detect wide range of edges in images. The canny operator works in a multi-stage process. Canny edge detection detects the edge of the object and in output it gives the outlined image.

\subsection{Morphological filtering \\ 2.4.1 Grayscale Dilation}

Grayscale dilation using a structuring element is performed on mammogram images which lead to bridge gaps.

\subsubsection{Hole-Filling}

In this step, the task of filling the gaps or holes in the image is performed. The dilated gradient mask shows the outline of the cell quite nicely, but there are still some holes in the interior of the tumor.

\subsubsection{Grayscale Erosion}

Grayscale erosion using a structuring element is applied on mammogram images which lead to obtain margin of the exact mass. So here the object is shrink to obtain a concise mass which gives an accurate idea about the mass.

\subsubsection{Suppress Light Structures Connected To Im- age Border}

After performing all these above tasks the final border is detected as shown in table 2. For displaying the segmented object an outline around the segmented tumor is placed. After that the original mammogram with outline is obtained.
Table 2. Morphological filtering on mammograms

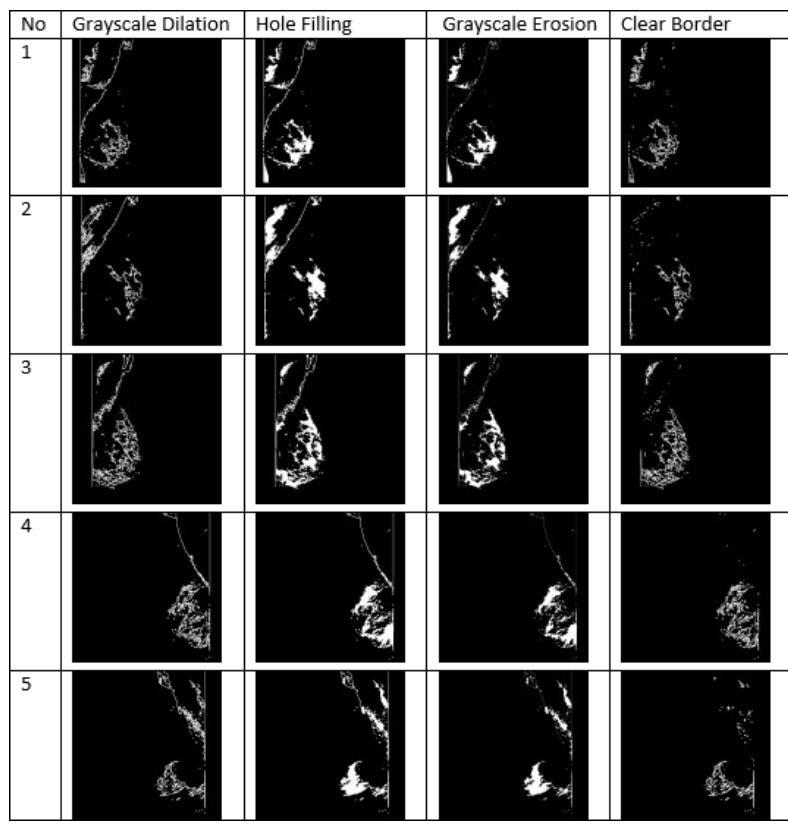

\subsection{Feature Selection}

\subsubsection{Original Mammogram with Border}

Overlap the original image with the eroded image and highlight the border in red to get a clear view of the edges on the masses as shown in figure 4.
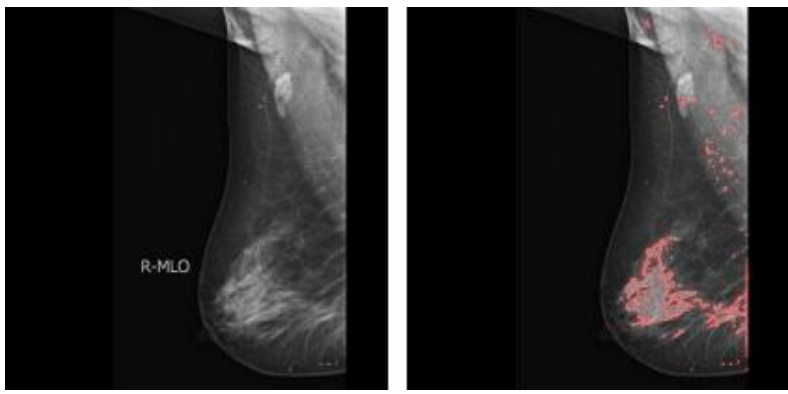

Fig. 4.(a) Original mammogram(b) Original mammogram with outline

\subsection{Region Growing Based Segmented Mass Classification}

2.6.1 Segmented mass classification

Classification of segmented mass can be done using various methods like classification of breast masses using selected shape, edge-sharpness, and texture features with linear and kernel-based classifiers, breast mass classification on sonographic images on the basis of shape analysis, adaptive Neuro-fuzzy inference system, area, bounding box, centroid, filled area, filled image, image, pixel Idx list, pixel list, and sub array Idx etc. Bounding box is used to classify the segmented mass as shown in figure 5 . 

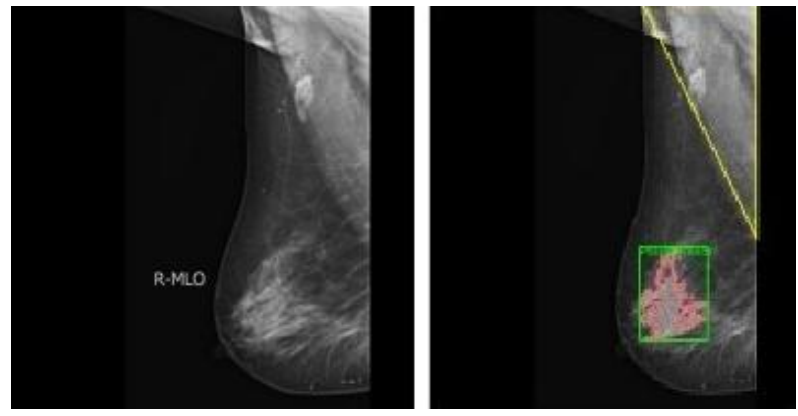

Fig. 5. (a) Original mammogram(b) Segmented mass classification

\section{EXPERIMENTAL RESULTS}

Table 3 contains two columns. The first column shows the results of the existing techniques [8]. The second column shows the results of the proposed scheme. Here a comparison is shown between the existing technique and the proposed scheme in the form of tabular data. The proposed approach is implemented in Matlab 13. Mammogram images have been collected from various hospitals. This approach has been successfully tested to detect possible lesion and pectoral muscle in more than 50 mammograms. Possible tumor portion in mammogram images are obtained as shown in table 4 .

Table 3. Comparison of existing technique with proposed scheme

\begin{tabular}{|l|c|c|}
\hline No & 1 & 2 \\
\hline $\begin{array}{l}\text { Patient } \\
\text { original } \\
\text { Mammogram }\end{array}$ & & \\
\hline $\begin{array}{l}\text { Mammogram } \\
\text { after } \\
\text { threshold }\end{array}$ & & \\
\hline $\begin{array}{l}\text { Mammogram } \\
\text { after edge } \\
\text { detection }\end{array}$ & \\
\hline
\end{tabular}

Table 4. Possible tumor in mammogram images of five patients

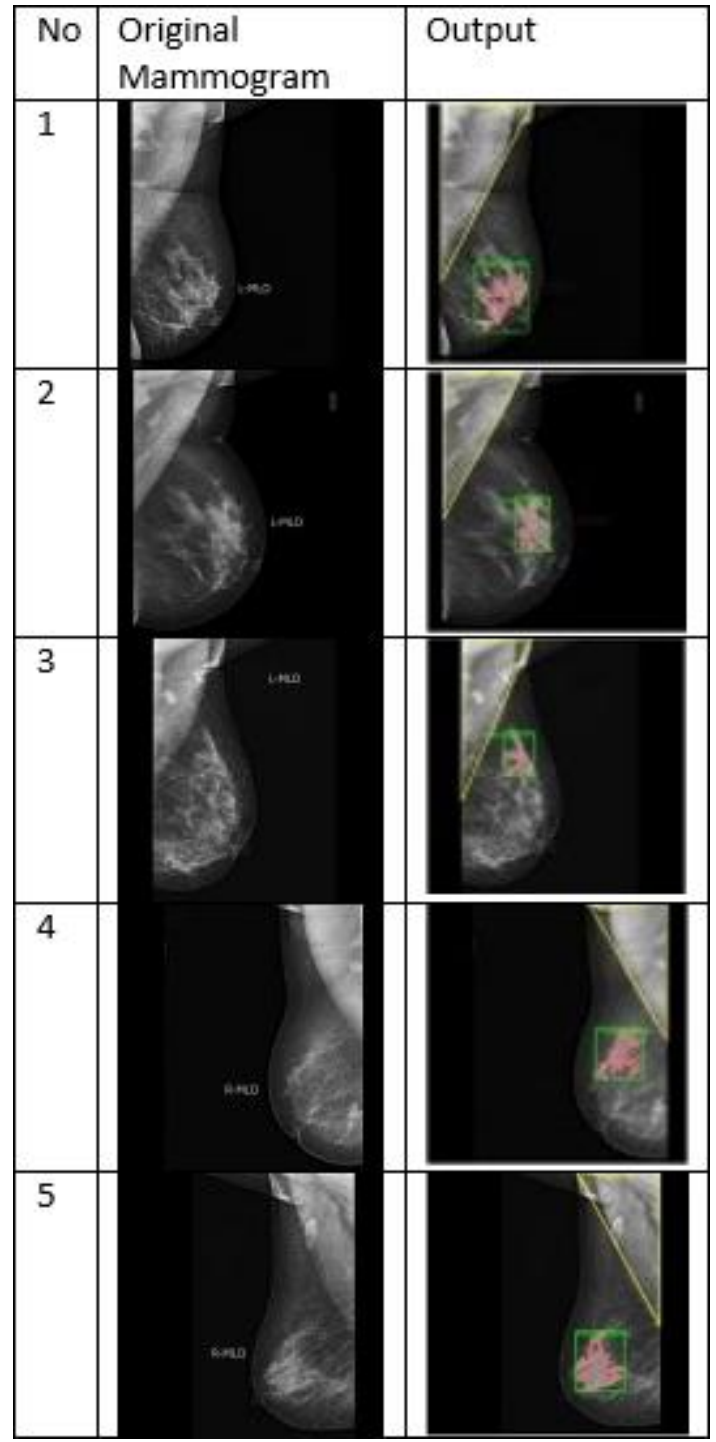

\section{CONCLUSION}

Pre-processing task is performed on the mammogram to remove the noise, labels and the pectoral muscle and focus on the region of interest. Then the mammogram is segmented so that only the masses are clearly seen in the mammogram. Edge detection is performed to get a border over the masses. To get distinct and clear masses morphological filtering is performed which includes grayscale dilation, hole-filling, grayscale erosion and clear border processes. Finally to highlight the possible lesion in the mammogram the processed mammogram image is superimposed on the original mammogram.

\section{FUTURE WORK}

Classification of mammogram images as normal or abnormal. If the mammogram is abnormal then classification is possible based on its shape like irregular, circumscribed and micro calcifications.

\section{ACKNOWLEDGMENTS}

Our sincere thanks to the Dr. Pankaj Shah, M.D. and Dr.Jigar Shah for guiding us and giving us their valuable suggestions. 


\section{REFERENCES}

[1] Spandana p, Rao, K.M.M., Rao, B.V.V.S.N., and Jwalasrikala J. 2013 "Novel image processing techniques for early detection of breast cancer, mat lab and lab view implementation." Date of Conference: 16-18 Jan. 2013, Bangalore, India, Pages 105 - 108.

[2] Woo Kyung Moon, Yi-Wei Shen, Min Sun Bae and Chiun-Sheng Huangand. 2013 "Computer-Aided Tumor Detection Based on Multi-Scale Blob Detection Algorithm in Automated Breast Ultrasound Images." Date of Conference: 27 June 2013, Medical Imaging, IEEE Transactions on (Volume: 32, Issue: 7), Pages 1191 1200 .

[3] Bozek J Mustra, M. and Grgic M. 2009 “A survey of mammographic image processing algorithms for bilateral asymmetry detection." Date of Conference: 28-30 Sept. 2009, ELMAR, 2009. ELMAR '09. International Symposium, Pages 9 - 14.

[4] Cahoon, T.C Sutton, M.A and Bezdek, J.C. 2000 "Breast cancer detection using image processing techniques." Date of Conference: 2000, Fuzzy Systems, 2000. The Ninth IEEE International Conference on (Volume: 2), Pages 973 - 976 vol.2.
[5] Kyoung-Mi Lee.2003 “An adaptive resource-allocating network for automated detection, segmentation, and classification of breast cancer nuclei topic area: image processing and recognition." Date of Conference: 28-30 Sept. 2009, Neural Networks, IEEE Transactions on (Volume: 14, Issue: 3), Pages 680 - 687.

[6] Mencattini A, Salmeri M., Lojacono R., Frigerio M. and Caselli F. 2008 "Mammographic Images Enhancement and Denoising for Breast Cancer Detection Using Dyadic Wavelet Processing." Date of Conference: 28 May 2008 (Volume: 57, Issue: 7), Pages 1422 - 1430.

[7] Vidhya M, Sangeetha, Vimalkumar and Helenprabha K. 2011 "Early stage detection of cancer in mammogram using statistical feature extraction." Date of Conference: 15-17 Dec. 2011, Advancements in Electrical, Electronics and Control Engineering (ICONRAEeCE), 2011 International Conference on, Pages 401 - 404.

[8] Al-Shamlan H and El-Zaart A. 2010 "Feature extraction values for breast cancer mammography images." Date of Conference: 16-18 April 2010, Bioinformatics and Biomedical Technology (ICBBT), 2010 International Conference on, Pages 335 - 340. 